\title{
Association between diabetes mellitus and anemia among Korean adults according to sex: a cross-sectional analysis of data from the Korea National Health and Nutrition Examination Survey (2010-2016)
}

\author{
Mihye Kim¹, Sook-Hyun Lee ${ }^{2}$, Kyoung Sun Park', Eun-Jung Kim³ ${ }^{3}$ Sujung Yeo ${ }^{4}$ and In-Hyuk Ha2*
}

\begin{abstract}
Background: There are many conflicting opinions regarding the association between anemia and diabetes mellitus $(D M)$, and the mechanism by which DM influences anemia remains uncertain. Therefore, we aimed to investigate the association between anemia and DM in Korean adults and to analyze the risk factors for anemia among these patients according to sex.

Methods: This retrospective cross-sectional survey was conducted using data from the Korea National Health and Nutrition Examination Survey V, VI, and VII between January 2010 and December 2016. In total, 25,597 Korean adults aged $\geq 19$ years (10,117 men, 15,480 women) were included. Patients with a fasting blood sugar level of $\geq 126 \mathrm{mg} /$ $\mathrm{dL}$ or who have been diagnosed with DM were classified as the DM group. Anemia was defined as hemoglobin levels of $<13 \mathrm{~g} / \mathrm{dL}$ in men and $<12 \mathrm{~g} / \mathrm{dL}$ in women. Logistic regression analysis was used to adjust for demographic characteristics and lifestyle-, disease-, and health-related factors.

Results: Approximately $11.3 \%$ of patients had DM. The prevalence of anemia was significantly higher in the DM group than in the non-DM group. After adjusting for confounding factors, the odds of the prevalence of anemia in men were higher in the DM group than in the non-DM group (odds ratio [OR] 1.87, 95\% confidence interval [Cl] $1.42-2.50, p<0.0001)$. When investigated according to the serum creatinine level, the association was significantly stronger among women (OR 42.63, 95\% Cl 17.25-105.36, $p<0.0001$ ) than among men (OR 6.30, 95\% Cl 3.08-12.90, $p<0.0001)$.
\end{abstract}

Conclusions: We found a strong association between DM and anemia that was more prominent among men than among women. We also determined that the serum creatinine level had a greater influence on DM and anemia in women than in men.

Keywords: Diabetes mellitus, Anemia, Cross-sectional study, Korea National Health and Nutrition Examination Survey

\footnotetext{
* Correspondence: hanihata@gmail.com

${ }^{2}$ Jaseng Spine and Joint Research Institute, Jaseng Medical Foundation, 3F JS Tower, 538 Gangnam-daero, Gangnam-gu, Seoul 06110, Republic of Korea Full list of author information is available at the end of the article
}

(c) The Author(s). 2021 Open Access This article is licensed under a Creative Commons Attribution 4.0 International License, which permits use, sharing, adaptation, distribution and reproduction in any medium or format, as long as you give appropriate credit to the original author(s) and the source, provide a link to the Creative Commons licence, and indicate if changes were made. The images or other third party material in this article are included in the article's Creative Commons licence, unless indicated otherwise in a credit line to the material. If material is not included in the article's Creative Commons licence and your intended use is not permitted by statutory regulation or exceeds the permitted use, you will need to obtain permission directly from the copyright holder. To view a copy of this licence, visit http://creativecommons.org/licenses/by/4.0/. The Creative Commons Public Domain Dedication waiver (http://creativecommons.org/publicdomain/zero/1.0/) applies to the data made available in this article, unless otherwise stated in a credit line to the data. 


\section{Background}

Diabetes mellitus (DM) is a chronic disease that causes hyperglycemia because of impaired insulin secretion by pancreatic beta cells and peripheral insulin resistance, which may lead to serious complications [1]. As of 2019, the number of patients with DM worldwide was approximately 422 million, with an estimated prevalence of $8.5 \%$ [2]. The incidence of DM is increasing most rapidly among middle-to-low income countries [3], and it is predicted that one in 10 adults aged $\geq 20$ years will have DM by 2045 [4]. Such a marked increase in the prevalence of DM could pose a significant economic burden on a country's healthcare system. According to data from the Health Insurance Review and Assessment Service [5], the total cost of care for patients diagnosed with DM in the US was 573.7 billion won in 2019, an increase of $8.3 \%$ from that in the previous year [6].

Approximately $50-80 \%$ of patients with cardiovascular disease die, and DM may increase the risk of cardiovascular disease by increasing blood cholesterol and triglyceride levels. Accordingly, cardiovascular disease is considered a major cause of premature death, and its incidence among patients with DM reaches $20 \%$ after approximately 7 years [7]. Moreover, DM is often accompanied by acute or chronic complications, and because chronic complications are directly associated with patient death, prevention of such complications is of utmost importance.

Anemia, which is a major global public health issue, is a disease caused by the reduced ability to transport oxygen to tissues due to the lack of hemoglobin $(\mathrm{Hb})$ [8]. Anemia is a common disease with a prevalence of nearly one-third of the world's population, including that in Korea $[9,10]$. It is more common among women than among men and in the older population than in the younger population [11]. The causes of anemia are complex and multifactorial; as it is a potential risk factor for cardiovascular disease, it may originate from complications related to various chronic diseases. However, there are many conflicting opinions about its association with DM.

Numerous studies on the association between DM and anemia have been published. Studies have demonstrated that anemia occurs in $14-45 \%$ of patients with DM [12, 13]. A study from the United States that analyzed adults aged $\geq 20$ years reported that there is no association between DM and the onset of anemia among men or women [14]. This review of previous studies indicates that there are conflicting views on the association between anemia and DM, and the mechanism by which DM influences anemia remains uncertain.

Accordingly, we aimed to investigate the association between DM and anemia among Korean adults using nationally representative data from the Korea National Health and Nutrition Examination Survey (KNHANES)
V, VI, and VII to analyze risk factors that could cause anemia in patients with DM. In particular, we sought to analyze sex-based differences in specific disease factors that could affect DM and anemia among Korean adults.

\section{Methods \\ Study design and population}

The present study used data from the KNHANES V, VI, and VII. The KNHANES is a cross-sectional survey conducted every 3 years to assess the health and nutritional status of the general Korean population. The survey includes a health screening, health questionnaire survey, and nutritional survey conducted with a nationally representative sample extracted by stratified, clustered, and systematic sampling methods [15].

The study population in the present study comprised adults aged $\geq 19$ years who took part in the health screening and health questionnaire survey among a sample population of 56,632 individuals who participated in the KNHANES, which was conducted between 2010 and 2016. The study population was limited by applying the following exclusion criteria: (a) age < 19 years ( $n=$ 12,617 ) and (b) missing values for anemia, hypertension, $\mathrm{DM}$, hypertriglyceridemia, iron intake, $\mathrm{Hb}$ level, or serum creatinine level; a low high-density lipoprotein (HDL) cholesterol level; and a high waist circumference. After excluding 31,035 participants from a total of 56,632 individuals, the final data set consisted of 25,597 (45.2\%) participants (Fig. 1).

\section{Outcomes and other variables Measurement of DM}

Based on the Korean Diabetes Association's clinical practice guidelines (2016) [16], individuals with a fasting blood sugar (FBS) level of $\geq 126 \mathrm{mg} / \mathrm{dL}$ or who responded as having been diagnosed with DM were classified as the DM group, and those with an FBS level of $<126 \mathrm{mg} / \mathrm{dL}$ or who had not been diagnosed with DM were classified as the non-DM group.

\section{Measurement of $\mathrm{Hb}$ levels}

$\mathrm{Hb}$ level was used as the indicator of anemia. Based on the criteria put forth by the World Health Organization [17], anemia was defined as $\mathrm{Hb}$ levels of $<13 \mathrm{~g} / \mathrm{dL}$ in men and $<12 \mathrm{~g} / \mathrm{dL}$ in women.

\section{Other covariates}

We assessed the following covariates: demographic (including sex), lifestyle, disease, and health-related factors. Age was treated as a continuous variable, whereas sex was divided into men and women. Average monthly household income was divided into quartiles: low, lower middle, higher middle, and high. Education level was divided into four categories: below primary school, middle 
KNHANES $\vee-1$ (2010): 8,958

KNHANES $V-2$ (2011): 8,518

KNHANES $V-3$ (2012): 8,058

KNHANES VI-1 (2013): 8,018

KNHANES VI-2 (2014): 7,550

KNHANES VI-3 (2015): 7,380

KNHANES VII-1 (2016): 8,150

Total $n=56,632$

Excluded those aged $<19$ years $(n=12,617)$

Excluded those with missing information in the

following areas:

- Anemia $(n=5,343)$

- Hypertension survey ( $n=4,509$ )

- Diabetes mellitus survey $(n=7,643)$

Hypertriglyceridemia survey $(n=13,582)$

- Low HDL survey $(n=5,212)$

- High waist circumference $(n=2,403)$

- Iron intake per day $(n=5,122)$

- Hemoglobin ( $n=5,343)$

- Serum creatinine ( $n=5,212)$

- Demographics ( $n=5,701$ )

Final analysis set ( $n=25,597)$

Diabetes mellitus $(n=2,903)$

Non-diabetes mellitus ( $n=22,694$ )

Fig. 1 Inclusion and exclusion criteria for selecting participants from the 2010-2016 KNHANES V-VII HDL: high-density lipoprotein; KNHANES, Korea National Health and Nutrition Examination Survey

school, high school, and college or higher. Body mass index (BMI) was calculated as weight divided by height squared; underweight, normal weight, and overweight or obesity were defined as BMI of $<18.5 \mathrm{~kg} / \mathrm{m}^{2}, 18.5-24.9$ $\mathrm{kg} / \mathrm{m}^{2}$, and $\geq 25.0 \mathrm{~kg} / \mathrm{m}^{2}$, respectively. Smoking status was divided into non-/ex-smoker and current smoker, and alcohol consumption was categorized as yes and no. For daily iron intake, data from the food-intake portion of the KNHANES were used, and the following criteria were applied: $10 \mathrm{mg}$ for men aged 19-64 years, $9 \mathrm{mg}$ for men aged $\geq 65$ years, $14 \mathrm{mg}$ for women aged 19-49 years, $8 \mathrm{mg}$ for women aged 50-74 years, and $7 \mathrm{mg}$ for women aged $\geq 75$ years [18]. All blood samples were collected from the participants under fasting and analyzed within $24 \mathrm{~h}$. Serum creatinine level and plasma glucose level were measured using hexokinase UV with a Hitachi 7600 Automatic Analyzer (Hitachi, Tokyo, Japan) [19]. The HbA1c level was analyzed using Tosoh G8 highperformance liquid chromatography (Tosoh, Tokyo, Japan). Hypertension was defined as a blood pressure of $>140 / 90 \mathrm{mmHg}$. Hypertriglyceridemia [20] was defined as a triglyceride level of $\geq 200 \mathrm{mg} / \mathrm{dL}$, which indicates classification into the high or very high group according to the National Cholesterol Education Program Adult Treatment Panel III (NCEP ATP III). Low HDL cholesterol levels were defined as $<40 \mathrm{mg} / \mathrm{dL}$ for men and $<$ $50 \mathrm{mg} / \mathrm{dL}$ for women.

\section{Statistical analysis}

Because the KNHANES contains data based on a complex sample design, complex-sample statistical analysis was used with consideration of weight, stratification variables, and cluster variables. Differences in the characteristics of the study population depending on DM and anemia were analyzed using the Rao-Scott chi-square test or t-test. Data of categorical variables are expressed as frequency and percentage (\%), whereas data of continuous variables are expressed as mean \pm standard error.

To analyze the associations with anemia depending on $\mathrm{DM}$, logistic regression analysis was performed to derive the odds ratio (OR) and confidence interval (CI). Logistic regression was used to assess the impact of DM, sex, and the interaction between DM and sex on developing anemia and to calculate OR. Additionally, subgroup analyses were performed on factors that may influence anemia in patients with DM by sex (men versus women). 
SAS, version 9.4 (SAS Institute Inc., Cary, NC), was used for all statistical analyses. The significance level was set to $p$-values of $<0.05$ for all tests.

\section{Results}

Table 1 shows the characteristics of the study population depending on DM. There were 2903 participants (11.3\%) in the DM group and 22,694 participants (88.7\%) in the non-DM group and more women $(n=1486)$ than men $(n=1417 ; 48.8 \%)$. Mean patient age in the DM group was $60.09 \pm 0.31$ years, which was higher than that in the non-DM group. With regard to education level, "below primary school" was the most common response $(n=$ 1299; 44.8\%) in the DM group, whereas "high school" was the most common response $(n=7668 ; 33.8 \%)$ in the non-DM group $(p<0.0001)$. Mean BMI $\left(\mathrm{kg} / \mathrm{m}^{2}\right)$ was higher in the DM group $(25.3 \pm 0.1)$ than in the non-DM group $(23.6 \pm 0.03)(p<0.0001)$; there were $1490(51.3 \%)$ participants with a normal weight in the DM group and 14,766 (65.1\%) in the non-DM group, showing higher percentages than both the underweight and overweight and obesity subgroups $(p<0.0001)$. There were more non-/ex-smokers in the DM group $(n=18,983 ; 83.7 \%)$ than in the non-DM group $(n=2352 ; 81 \%)(p=0.0076)$. With regard to alcohol consumption, the number of participants who responded "yes" was 2284 (78.7\%) in the DM group and 19,781 (87.2\%) in the non-DM group, which was much higher than the number of participants who responded "no" $(p<0.0001)$.

As for clinical factors that influence DM, the DM group showed a higher percentage and mean value for waist circumference, a high waist circumference, FBS level $(\mathrm{mg} / \mathrm{dL}), \mathrm{HbA1c}$ level, hypertension, hypertriglyceridemia, and a low HDL cholesterol level than the nonDM group $(p<0.0001)$. However, there were no significant differences in daily iron intake or $\mathrm{Hb}$ level.

Table 2 shows the characteristics of the study population depending on diabetes and anemia. There were 2258 participants $(8.9 \%)$ in the anemia group and 23,339 $(91.1 \%)$ in the non-anemia group, and there were more women $(n=1796,79.5 \%)$ than men in the anemia group $(n=462,20.5 \%)(p<0.0001)$. Mean patient age in the anemia group with DM was $65.93 \pm 0.86$ years, which was higher than that in the non-anemia group in DM and all groups in non-DM $(p<0.0001)$. Regarding household income, the prevalence of anemia tended to be higher among the lower income quartiles in the DM group, whereas it tended to increase with an increasing income level in the non-DM group.

With regard to education level in the non-DM group, "high school" was the most common response in both the anemia $(n=678,35.1 \%)$ and non-anemia $(n=6990$, $33.7 \%)$ groups $(p<0.0001)$. In contrast, the most common education level in the DM group was " $\leq$ Elementary
Table 1 Characteristics of the study population with and without diabetes mellitus

\begin{tabular}{|c|c|c|c|}
\hline Variables & $\begin{array}{l}\text { Non-DM } \\
(n=22,694)\end{array}$ & $\begin{array}{l}\text { DM } \\
(n=2903)\end{array}$ & $p$-value \\
\hline Age (years) ${ }^{a}$ & $45.28 \pm 0.13$ & $60.09 \pm 0.31$ & $<.0001$ \\
\hline \multicolumn{4}{|l|}{ Sex ${ }^{b}$} \\
\hline Men & $8700(38.3)$ & $1417(48.8)$ & \multirow[t]{2}{*}{$<.0001$} \\
\hline Women & $13994(61.7)$ & $1486(51.2)$ & \\
\hline \multicolumn{4}{|l|}{ Household income ${ }^{b}$} \\
\hline Low & $4082(18)$ & $1013(34.9)$ & \multirow[t]{4}{*}{$<.0001$} \\
\hline Lower middle & $5780(25.5)$ & $796(27.4)$ & \\
\hline Higher middle & $6380(28.1)$ & $588(20.3)$ & \\
\hline High & $6452(28.4)$ & $506(17.4)$ & \\
\hline \multicolumn{4}{|l|}{ Educational level ${ }^{b}$} \\
\hline$\leq$ Elementary school & $5168(22.8)$ & $1299(44.8)$ & \multirow[t]{4}{*}{$<.0001$} \\
\hline Middle school & $2320(10.2)$ & $449(15.5)$ & \\
\hline High school & 7668 (33.8) & $750(25.8)$ & \\
\hline$\geq$ College & 7538 (33.2) & $405(14)$ & \\
\hline $\mathrm{BMI}(\mathrm{kg} / \mathrm{m} 2)^{\mathrm{a}, \mathrm{b}}$ & $23.6 \pm 0.03$ & $25.27 \pm 0.09$ & $<.0001$ \\
\hline < 18.5 (underweight) & $1005(4.4)$ & $36(1.2)$ & \multirow[t]{3}{*}{$<.0001$} \\
\hline < 25 (normalweight) & $14766(65.1)$ & $1490(51.3)$ & \\
\hline$\geq 25$ (overweight or obese) & $6923(30.5)$ & $1377(47.4)$ & \\
\hline \multicolumn{4}{|l|}{ Smoking status ${ }^{b}$} \\
\hline Non-/Ex-smoker & $18,983(83.7)$ & $2352(81)$ & \multirow[t]{2}{*}{0.0076} \\
\hline Current smoker & $3711(16.4)$ & $551(19)$ & \\
\hline \multicolumn{4}{|l|}{ Alcohol consumption ${ }^{b}$} \\
\hline No & $2913(12.8)$ & $619(21.3)$ & \multirow[t]{2}{*}{$<.0001$} \\
\hline Yes & $19,781(87.2)$ & $2284(78.7)$ & \\
\hline Non-Anemia & $20,764(91.5)$ & $2575(88.7)$ & $<.0001$ \\
\hline Anemia & $1930(8.5)$ & $328(11.3)$ & $<.0001$ \\
\hline Iron intake per day (mg/day) ${ }^{a}$ & $16.68 \pm 0.16$ & $16.66 \pm 0.38$ & 0.9651 \\
\hline Hemoglobin (g/dL) ${ }^{\text {a }}$ & $14.12 \pm 0.01$ & $14.14 \pm 0.04$ & 0.6599 \\
\hline Serum creatinine $(\mathrm{mg} / \mathrm{dL})^{\text {a }}$ & $0.83 \pm 0.00$ & $0.9 \pm 0.01$ & $<.0001$ \\
\hline Waist circumference $(\mathrm{cm})^{a}$ & $80.56 \pm 0.08$ & $87.53 \pm 0.23$ & $<.0001$ \\
\hline High waist circumference ${ }^{b}$ & $5481(24.2)$ & $1360(46.9)$ & $<.0001$ \\
\hline $\mathrm{HTN}^{\mathrm{b}}$ & $6487(28.6)$ & $1822(62.8)$ & $<.0001$ \\
\hline Fasting glucose level $(\mathrm{mg} / \mathrm{dL})^{a}$ & $92.96 \pm 0.08$ & $142.84 \pm 1.01$ & $<.0001$ \\
\hline $\operatorname{HbA1c}(\%)^{a^{\prime}}$ & $5.53 \pm 0.00$ & $7.36 \pm 0.04$ & $<.0001$ \\
\hline Hypertriglyceridemia ${ }^{\text {b }}$ & $2924(12.9)$ & $777(26.8)$ & $<.0001$ \\
\hline Low HDL cholesterol ${ }^{\mathrm{b}}$ & $8151(35.9)$ & $1562(53.8)$ & $<.0001$ \\
\hline
\end{tabular}

a Continuous variables are presented means \pm standard error and compared using t-test

${ }^{\mathrm{b}}$ Categorical variables are presented as frequencies and percentages and compared using the Rao-Scott Chi-Square test

DM diabetes mellitus $\boldsymbol{B} \boldsymbol{M} \boldsymbol{I}$ body mass index; $\boldsymbol{H T N}$ hypertension; $\boldsymbol{H} \boldsymbol{B} \boldsymbol{A} \mathbf{C} \boldsymbol{C}$ glycated hemoglobin; HDL high-density lipoprotein 
Table 2 Characteristics according to with or without of diabetes and anemia

\begin{tabular}{|c|c|c|c|c|c|c|}
\hline \multirow[t]{2}{*}{ Variables } & \multicolumn{3}{|l|}{ Non-DM } & \multicolumn{3}{|l|}{ DM } \\
\hline & $\begin{array}{l}\text { Non-anemia } \\
(n=20,764)\end{array}$ & $\begin{array}{l}\text { Anemia } \\
(n=1930)\end{array}$ & $p$-value & $\begin{array}{l}\text { Non-anemia } \\
(n=2575)\end{array}$ & $\begin{array}{l}\text { Anemia } \\
(n=328)\end{array}$ & $p$-value \\
\hline Age (years) ${ }^{a}$ & $45.08 \pm 0.18$ & $47.57 \pm 0.46$ & $<.0001$ & $59.36 \pm 0.33$ & $65.93 \pm 0.86$ & $<.0001$ \\
\hline \multicolumn{7}{|l|}{ Sex ${ }^{b}$} \\
\hline Men & $8376(40.3)$ & $324(16.8)$ & $<.0001$ & $1279(49.7)$ & $138(42.1)$ & $<.0001$ \\
\hline Women & $12388(59.7)$ & 1606 (83.2) & & $1296(50.3)$ & $190(57.9)$ & \\
\hline \multicolumn{7}{|l|}{ Household income ${ }^{b}$} \\
\hline Low & 3661 (17.6) & $421(21.8)$ & $<.0001$ & $876(34.0)$ & $137(41.8)$ & 0.0095 \\
\hline Lower middle & $5271(25.4)$ & $509(26.4)$ & & $705(27.4)$ & $91(27.7)$ & \\
\hline Higher middle & $5854(28.2)$ & $526(27.3)$ & & $537(20.9)$ & $51(15.6)$ & \\
\hline High & $5978(28.8)$ & $474(24.6)$ & & $457(17.8)$ & $49(14.9)$ & \\
\hline \multicolumn{7}{|l|}{ Educational level ${ }^{b}$} \\
\hline$\leq$ Elementary school & $4671(22.5)$ & $497(25.8)$ & $<.0001$ & $1118(43.4)$ & $181(55.2)$ & $<.0001$ \\
\hline Middle school & $2160(10.4)$ & $160(8.3)$ & & $411(16.0)$ & $38(11.6)$ & \\
\hline High school & 6990 (33.7) & $678(35.1)$ & & $672(26.1)$ & 78 (23.8) & \\
\hline$\geq$ College & $6943(33.4)$ & $595(30.8)$ & & $374(14.5)$ & $31(9.5)$ & \\
\hline BMI $\left(\mathrm{kg} / \mathrm{m}^{2}\right)^{\mathrm{a}, \mathrm{b}}$ & $23.67 \pm 0.03$ & $22.68 \pm 0.09$ & $<.0001$ & $25.37 \pm 0.09$ & $24.46 \pm 0.42$ & $<.0001$ \\
\hline < 18.5 (underweight) & $864(4.2)$ & $141(7.3)$ & $<.0001$ & $22(0.9)$ & $14(4.3)$ & $<.0001$ \\
\hline$<25$ (normoweight) & $13,409(64.6)$ & $1357(70.3)$ & & $1294(50.3)$ & $196(59.8)$ & \\
\hline$\geq 25$ (overweight or obese) & $6491(31.3)$ & $432(22.4)$ & & $1259(48.9)$ & $118(36)$ & \\
\hline \multicolumn{7}{|l|}{ Smokers ${ }^{b}$} \\
\hline Non-/Ex-smoker & $17151(82.6)$ & $1832(94.9)$ & $<.0001$ & $2066(80.2)$ & $286(87.2)$ & 0.0001 \\
\hline Current smoker & $3613(17.4)$ & $98(5.1)$ & & $509(19.8)$ & $42(12.8)$ & \\
\hline \multicolumn{7}{|l|}{ Alcohol consumption ${ }^{\text {b }}$} \\
\hline No & $2598(12.5)$ & $315(16.3)$ & $<.0001$ & $533(20.7)$ & $86(26.2)$ & 0.0068 \\
\hline Yes & $18166(87.5)$ & $1615(83.7)$ & & $2042(79.3)$ & $242(73.8)$ & \\
\hline Iron intake per day $\left(\mathrm{mg} /\right.$ day) ${ }^{a}$ & $16.19 \pm 0.17$ & $14.06 \pm 0.26$ & $<.0001$ & $16.31 \pm 0.43$ & $12.68 \pm 0.52$ & $<.0001$ \\
\hline Hemoglobin $(\mathrm{g} / \mathrm{dL})^{a}$ & $14.37 \pm 0.01$ & $11.02 \pm 0.03$ & $<.0001$ & $14.47 \pm 0.03$ & $11.31 \pm 0.07$ & $<.0001$ \\
\hline Serum creatinine $(\mathrm{mg} / \mathrm{dL})^{a}$ & $0.83 \pm 0.002$ & $0.8 \pm 0.02$ & $<.0001$ & $0.87 \pm 0.005$ & $1.23 \pm 0.13$ & $<.0001$ \\
\hline Waist circumference $(\mathrm{cm})^{a}$ & $80.85 \pm 0.1$ & $76.97 \pm 0.26$ & $<.0001$ & $87.85 \pm 0.24$ & $84.82 \pm 0.88$ & $<.0001$ \\
\hline High waist circumference ${ }^{b}$ & $5151(24.8)$ & $330(17.1)$ & $<.0001$ & $1242(48.2)$ & $118(36.0)$ & 0.0003 \\
\hline Fasting glucose level (mg/dL) ${ }^{a}$ & $93.09 \pm 0.1$ & $91.3 \pm 0.24$ & $<.0001$ & $144.13 \pm 1.05$ & $131.83 \pm 3.09$ & $<.0001$ \\
\hline HTN $^{b}$ & $5967(28.7)$ & $520(26.9)$ & 0.1012 & $1584(61.5)$ & $238(72.6)$ & 0.0008 \\
\hline $\operatorname{HbA1c}(\%)^{a}$ & $5.52 \pm 0.005$ & $5.62 \pm 0.01$ & $<.0001$ & $7.37 \pm 0.04$ & $7.28 \pm 0.1$ & $<.0001$ \\
\hline Hypertriglyceridemia ${ }^{\text {b }}$ & 2816 (13.6) & $108(5.6)$ & $<.0001$ & 719 (27.9) & $58(17.7)$ & $<.0001$ \\
\hline Low HDL cholesterol ${ }^{b}$ & 7345 (35.4) & $806(41.8)$ & $<.0001$ & $1366(53.1)$ & $196(59.8)$ & 0.017 \\
\hline
\end{tabular}

${ }^{a}$ Continuous variables are presented means \pm standard error and compared using t-test

${ }^{b}$ Categorical variables are presented as frequencies and percentages and compared using the Rao-Scott Chi-Square test

$\boldsymbol{B} \boldsymbol{M I}$ body mass index; $\boldsymbol{H T N}$ hypertension; DM diabetes mellitus; $\boldsymbol{H} \boldsymbol{b A 1 \boldsymbol { c }}$ glycated hemoglobin; $\boldsymbol{H D L}$ high-density lipoprotein

school" in both the anemia $(n=181,55.2 \%)$ and nonanemia $(n=1118,43.4 \%)$ groups $(p<0.0001)$. Mean BMI $\left(\mathrm{kg} / \mathrm{m}^{2}\right)$ was higher in the non-anemia group than in the anemia group in both the non-DM and DM groups $(p<$ 0.0001 ). In the anemia group, the proportion of current smokers in the DM group (12.8\%) was relatively higher than that in the non-DM group $(5.1 \%)(p<0.0001)$.
Regarding clinical factors that influence anemia, there were significant differences in the daily iron intake $(\mathrm{mg} /$ day), $\mathrm{Hb}$ level $(\mathrm{g} / \mathrm{dL})$, serum creatinine level $(\mathrm{mg} / \mathrm{dL})$, waist circumference, high waist circumference, and fasting glucose level $(\mathrm{mg} / \mathrm{dL})$ between the two groups.

Table 3 shows the characteristics of the study population depending on anemia by sex in DM. Among men, 
Table 3 Characteristics of the study population with and without anemia according to sex in diabetes mellitus

\begin{tabular}{|c|c|c|c|c|c|c|}
\hline \multirow[t]{2}{*}{ Variables } & \multicolumn{3}{|l|}{ Men } & \multicolumn{3}{|l|}{ Women } \\
\hline & $\begin{array}{l}\text { Non-anemia } \\
(n=1279)\end{array}$ & $\begin{array}{l}\text { Anemia } \\
(n=138)\end{array}$ & $p$-value & $\begin{array}{l}\text { Non-anemia } \\
(n=1296)\end{array}$ & $\begin{array}{l}\text { Anemia } \\
(n=190)\end{array}$ & $p$-value \\
\hline Age (years) ${ }^{a}$ & $57.23 \pm 0.42$ & $68.09 \pm 0.91$ & $<.0001$ & $61.91 \pm 0.45$ & $64.58 \pm 1.28$ & $<.0001$ \\
\hline \multicolumn{7}{|l|}{ Household income $^{b}$} \\
\hline Low & $371(29)$ & $56(40.6)$ & 0.0031 & $505(39)$ & $81(42.6)$ & 0.4453 \\
\hline Lower middle & $350(27.4)$ & $39(28.3)$ & & $355(27.4)$ & $52(27.4)$ & \\
\hline Higher middle & $284(22.2)$ & $24(17.4)$ & & $253(19.5)$ & $27(14.2)$ & \\
\hline High & $274(21.4)$ & 19 (13.8) & & $183(14.1)$ & $30(15.8)$ & \\
\hline \multicolumn{7}{|l|}{ Educational level ${ }^{\mathrm{b}}$} \\
\hline$\leq$ Elementary school & $357(27.9)$ & $47(34.1)$ & 0.0075 & $761(58.7)$ & $134(70.5)$ & 0.2327 \\
\hline Middle school & $235(18.4)$ & $13(9.4)$ & & $176(13.6)$ & $25(13.2)$ & \\
\hline High school & 407 (31.8) & $55(39.9)$ & & $265(20.5)$ & $23(12.1)$ & \\
\hline$\geq$ College & $280(21.9)$ & $23(16.7)$ & & $94(7.3)$ & $8(4.2)$ & \\
\hline $\operatorname{BMI}\left(\mathrm{kg} / \mathrm{m}^{2}\right)^{\mathrm{a}, \mathrm{b}}$ & $25.1 \pm 0.12$ & $23.2 \pm 0.31$ & $<.0001$ & $25.69 \pm 0.13$ & $25.24 \pm 0.64$ & $<.0001$ \\
\hline$<18.5$ (underweight) & $8(0.6)$ & $8(5.8)$ & $<.0001$ & $14(1.1)$ & $6(3.2)$ & 0.105 \\
\hline$<25$ (normoweight) & $693(54.2)$ & $101(73.2)$ & & $601(46.4)$ & $95(50)$ & \\
\hline$\geq 25$ (overweight or obese) & $578(45.2)$ & $29(21)$ & & $681(52.6)$ & $89(46.8)$ & \\
\hline \multicolumn{7}{|l|}{ Smokers ${ }^{b}$} \\
\hline Non-/Ex-smoker & $825(64.5)$ & $106(76.8)$ & 0.0008 & $1241(95.8)$ & $180(94.7)$ & 0.6657 \\
\hline Current smoker & $454(35.5)$ & $32(23.2)$ & & $55(4.2)$ & $10(5.3)$ & \\
\hline \multicolumn{7}{|l|}{ Alcohol consumption ${ }^{\text {b }}$} \\
\hline No & $81(6.3)$ & $12(8.7)$ & 0.1235 & $452(34.9)$ & $74(39)$ & 0.4257 \\
\hline Yes & $1198(93.7)$ & $126(91.3)$ & & $844(65.1)$ & $116(61.1)$ & \\
\hline Iron intake per day (mg/day) a & $18.09 \pm 0.68$ & $14.05 \pm 0.61$ & $<.0001$ & $14.18 \pm 0.43$ & $11.83 \pm 0.78$ & $<.0001$ \\
\hline Hemoglobin $(\mathrm{g} / \mathrm{dL})^{a}$ & $15.27 \pm 0.04$ & $11.79 \pm 0.12$ & $<.0001$ & $13.51 \pm 0.03$ & $11.01 \pm 0.09$ & $<.0001$ \\
\hline Serum creatinine $(\mathrm{mg} / \mathrm{dL})^{a}$ & $0.98 \pm 0.01$ & $1.68 \pm 0.32$ & $<.0001$ & $0.73 \pm 0$ & $0.95 \pm 0.04$ & $<.0001$ \\
\hline Waist circumference $(\mathrm{cm})^{a}$ & $88.83 \pm 0.32$ & $84.75 \pm 0.92$ & $<.0001$ & $86.68 \pm 0.33$ & $84.86 \pm 1.32$ & $<.0001$ \\
\hline High waist circumference ${ }^{b}$ & $540(42.2)$ & $34(24.6)$ & 0.0002 & $702(54.2)$ & $84(44.2)$ & 0.0068 \\
\hline HTN ${ }^{b}$ & $743(58.1)$ & $98(71)$ & $<.0001$ & $841(64.9)$ & $140(73.7)$ & 0.3057 \\
\hline Fasting glucose level (mg/dL) ${ }^{a}$ & $147.17 \pm 1.55$ & $124.51 \pm 3.24$ & $<.0001$ & $140.5 \pm 1.48$ & $136.41 \pm 4.53$ & $<.0001$ \\
\hline $\mathrm{HbA1c}(\%)^{a}$ & $7.4 \pm 0.06$ & $7.08 \pm 0.12$ & $<.0001$ & $7.33 \pm 0.05$ & $7.41 \pm 0.13$ & $<.0001$ \\
\hline Hypertriglyceridemia ${ }^{\text {b }}$ & $417(32.6)$ & $19(13.8)$ & $<.0001$ & $302(23.3)$ & $39(20.5)$ & 0.6261 \\
\hline Low HDL cholesterol ${ }^{\text {b }}$ & 519 (40.6) & 61 (44.2) & 0.4338 & 847 (65.4) & 135 (71.1) & 0.3557 \\
\hline
\end{tabular}

a Continuous variables are presented means + standard error and compared using t-test

${ }^{b}$ Categorical variables are presented as frequencies and percentages and compared using the Rao-Scott Chi-Square test

$\boldsymbol{B M I}$ body mass index; $\boldsymbol{H T N}$ hypertension; $\boldsymbol{D} \boldsymbol{M}$ diabetes mellitus; $\boldsymbol{H} \boldsymbol{b A 1 \boldsymbol { c }}$ glycated hemoglobin; HDL, high-density lipoprotein

there were significant differences between the anemia and non-anemia groups except in alcohol consumption and low HDL cholesterol factors. Among women, there were significant differences between the groups in age, mean $\mathrm{BMI}$, daily iron intake, $\mathrm{Hb}$ level, serum creatinine level, waist circumference, high waist circumference, fasting glucose level, and $\mathrm{HbA1c}$ level.

Table 4 shows the association between DM and anemia analyzed using logistic regression analyses by sex; the results were significant in both the unadjusted model (Model 1) and adjusted models (Models 2 and 3) for men. In particular, the ORs for anemia in the DM group were higher than those in the non-DM group by 3.49-fold (95\% CI 2.69-4.53, $p<0.0001$ ), 2.18 -fold (95\% CI $1.67-2.85, p<0.0001$ ), and 1.87 -fold (95\% CI $1.42-$ 2.50, $p<0.0001$ ) in Models 1, 2, and 3, respectively. For women, the results were not significant in the unadjusted model (Model 1), although they were significant in the adjusted models (Models 2 and 3). In particular, the ORs for anemia were higher in the DM group than 
Table 4 Logistic regression analyses of the association between anemia and DM by sex

\begin{tabular}{|c|c|c|c|c|c|c|c|c|c|}
\hline & \multicolumn{3}{|c|}{ Model 1} & \multirow{2}{*}{$\begin{array}{l}\text { Model } 2 \\
\text { OR }^{a}\end{array}$} & \multicolumn{5}{|l|}{ Model 3} \\
\hline & $\mathrm{OR}^{\mathrm{a}}$ & $95 \% \mathrm{Cl}$ & $p$-value & & $95 \% \mathrm{Cl}$ & $p$-value & $\mathrm{OR}^{\mathrm{a}}$ & $95 \% \mathrm{Cl}$ & $p$-value \\
\hline \multicolumn{10}{|l|}{ Men } \\
\hline Non-DM & 1 & & & 1 & & & 1 & & \\
\hline DM & 3.49 & $2.69-4.53$ & $<.0001$ & 2.18 & $1.67-2.85$ & $<.0001$ & 1.87 & $1.42-2.50$ & $<.0001$ \\
\hline \multicolumn{10}{|l|}{ Women } \\
\hline Non-DM & 1 & & & 1 & & & 1 & & \\
\hline $\mathrm{DM}$ & 1.21 & $0.99-1.48$ & 0.0611 & 1.31 & $1.07-1.60$ & 0.0093 & 1.33 & $1.09-1.63$ & 0.0067 \\
\hline
\end{tabular}

Model 1: unadjusted

Model 2: adjusted for age, sex, household income, educational level, body mass index, smoking status, and alcohol consumption

Model 3: adjusted for age, sex, household income, educational level, body mass index, smoking status, alcohol consumption, iron intake per day, serum creatinine level, high waist circumference, hypertension, hypertriglyceridemia, and low high-density lipoprotein cholesterol level

a Odds ratios with adjustments using logistic regression models

DM diabetes mellitus; $\boldsymbol{O} \boldsymbol{R}$ odds ratio; $\mathbf{C l}$ confidence interval

those in the non-DM group by 1.31-fold (95\% CI 1.071.60, $p=0.0093)$ and 1.33 -fold (95\% CI 1.09-1.63, $p=$ 0.0067 ) in Models 2 and 3, respectively. Compared to the group without a history of DM, OR for the incident DM was significantly higher in an unadjusted model
(OR: 3.49; 95\% CI: 2.69-4.53); the association observed remained significant in Model 2 (OR: 3.10; 95\% CI: 2.38-4.04) and Model 3 (OR: 2.97; 95\% CI: 2.27-3.89). Among men (the reference category for sex), compared to the group without a history of DM, OR for the

Table 5 Odds ratios for risk factors of anemia in patients with diabetes by sex

\begin{tabular}{|c|c|c|c|c|c|c|c|c|c|}
\hline & \multicolumn{2}{|c|}{ Model 1} & \multirow[b]{2}{*}{$p$-value } & \multicolumn{2}{|c|}{ Model 2} & \multirow[b]{2}{*}{$p$-value } & \multirow{2}{*}{$\begin{array}{l}\text { Model } 3 \\
\text { OR }^{a}\end{array}$} & \multirow[b]{2}{*}{$95 \% \mathrm{Cl}$} & \multirow[b]{2}{*}{$p$-value } \\
\hline & $\mathrm{OR}^{\mathrm{a}}$ & $95 \% \mathrm{Cl}$ & & $\mathrm{OR}^{\mathrm{a}}$ & $95 \% \mathrm{Cl}$ & & & & \\
\hline \multicolumn{10}{|l|}{ Total } \\
\hline Iron intake per day (mg/day) & 0.96 & $0.95-0.98$ & $<.0001$ & 0.98 & $0.96-0.99$ & 0.0029 & 0.98 & $0.97-1.00$ & 0.0137 \\
\hline Serum creatinine (mg/dL) & 6.28 & $3.95-9.99$ & $<.0001$ & 14.28 & $8.15-25.01$ & $<.0001$ & 13.40 & $7.64-23.51$ & $<.0001$ \\
\hline High waist circumference & 0.59 & $0.44-0.79$ & 0.0004 & 0.52 & $0.31-0.88$ & 0.015 & 0.47 & $0.26-0.83$ & 0.0097 \\
\hline HTN & 1.69 & $1.25-2.29$ & 0.0007 & 1.30 & $0.94-1.79$ & 0.1133 & 1.09 & $0.78-1.53$ & 0.6134 \\
\hline Hypertriglyceridemia & 0.49 & $0.34-0.70$ & $<.0001$ & 0.69 & $0.42-0.90$ & 0.0129 & 0.62 & $0.41-0.92$ & 0.0179 \\
\hline Low HDL cholesterol & 1.41 & $1.064-1.87$ & 0.017 & 1.23 & $0.92-1.65$ & 0.1592 & 1.27 & $0.93-1.73$ & 0.1307 \\
\hline \multicolumn{10}{|l|}{ Men } \\
\hline Iron intake per day (mg/day) & 0.96 & $0.94-0.98$ & 0.0005 & 0.98 & $0.96-1.00$ & 0.02 & 0.98 & $0.96-1.00$ & 0.0744 \\
\hline Serum creatinine (mg/dL) & 10.31 & $5.40-19.70$ & $<.0001$ & 8.00 & $3.84-16.65$ & $<.0001$ & 6.30 & $3.08-12.90$ & $<.0001$ \\
\hline High waist circumference & 0.42 & $0.26-0.67$ & 0.0003 & 0.73 & $0.37-1.44$ & 0.3597 & 0.65 & $0.31-1.35$ & 0.2458 \\
\hline HTN & 2.49 & $1.61-3.85$ & $<.0001$ & 2.14 & $1.31-3.49$ & 0.0025 & 1.86 & $1.18-3.13$ & 0.0191 \\
\hline Hypertriglyceridemia & 0.24 & $0.13-0.43$ & $<.0001$ & 0.38 & $0.20-0.72$ & 0.003 & 0.40 & $0.21-0.77$ & 0.0065 \\
\hline Low HDL cholesterol & 1.17 & $0.79-1.73$ & 0.4322 & 1.32 & $0.85-2.06$ & 0.2178 & 1.34 & $0.88-2.20$ & 0.2462 \\
\hline \multicolumn{10}{|l|}{ Women } \\
\hline Iron intake per day (mg/day) & 0.98 & $0.95-1.00$ & 0.042 & 0.98 & $0.96-1.00$ & 0.0451 & 0.98 & $0.96-1.00$ & 0.1147 \\
\hline Serum creatinine (mg/dL) & 34.50 & $13.98-85.12$ & $<.0001$ & 37.12 & $15.35-89.76$ & $<.0001$ & 42.63 & $17.25-105.36$ & $<.0001$ \\
\hline High waist circumference & 0.61 & $0.42-0.89$ & 0.01 & 0.49 & $0.28-0.87$ & 0.0149 & 0.38 & $0.21-0.71$ & 0.0022 \\
\hline HTN & 1.25 & $0.83-1.86$ & 0.2835 & 1.06 & $0.70-1.60$ & 0.7868 & 0.89 & $0.58-1.35$ & 0.5721 \\
\hline Hypertriglyceridemia & 0.89 & $0.56-1.40$ & 0.6152 & 0.89 & $0.56-1.41$ & 0.6096 & 0.86 & $0.52-1.42$ & 0.5518 \\
\hline Low HDL cholesterol & 1.22 & $0.81-1.85$ & 0.349 & 1.20 & $0.81-1.77$ & 0.3736 & 1.22 & $0.80-1.86$ & 0.3684 \\
\hline
\end{tabular}

Model 1: unadjusted

Model 2: adjusted for age, sex, household income, educational level, body mass index, smoking status, and alcohol consumption

Model 3: adjusted for age, sex, household income, educational level, body mass index, smoking status, alcohol consumption, iron intake per day, serum creatinine level, high waist circumference, hypertension, hypertriglyceridemia, and low HDL cholesterol level

${ }^{\text {a }}$ Odds ratios with adjustments using logistic regression models

HTN hypertension; $\boldsymbol{H D L}$ high-density lipoprotein; $\boldsymbol{O}$ odds ratio; $\mathbf{C l}$ confidence interval 
incident DM was significantly higher in an unadjusted model (OR: 3.49; 95\% CI: 2.69-4.53); the association observed remained significant in Model 2 (OR: 3.10; 95\% CI: 2.38-4.04) and Model 3 (OR: 2.97; 95\% CI: $2.27-3.89)$.

Moreover, among those with no DM (the reference category for diabetes), men had a 5.52-fold higher OR for anemia (95\% CI 4.73-6.45, $p<0.0001$ ) in Model 1 , 4.36-fold higher OR (95\% CI 3.70-5.14, $p<0.0001)$ in Model 2, 5.66-fold higher OR (95\% CI 4.61-6.95, $p<$ $0.0001)$ in Model 3 than women.

Lastly, our study demonstrated a significant association in the interaction between sex and DM on the development of anemia in Model 1 (OR: 0.35; 95\% CI: $0.25-0.49, p<0.0001$ ), Model 2 (OR: 0.37; 95\% CI: 0.270.52, $p<0.0001$ ), and Model 3 (OR: 0.39; 95\% CI: $0.27-$ $0.54, p<0.0001$ ) in Additional file 1 . This can be interpreted as the odds for anemia for those with DM versus no DM being higher for men (the reference category for sex) than for women.

Table 5 shows the results of logistic regression analyses on regarding the influence of risk factors on the prevalence of anemia among patients with DM as the change in the odds ratio for a unit change in the continuous variables. The results were significant for daily iron intake, serum creatinine level, high waist circumference, and hypertriglyceridemia in Models 1, 2, and 3. In particular, the serum creatinine level showed ORs of 6.28 (95\% CI 3.95-9.99, $p<0.0001), 14.28$ (95\% CI 8.1525.01, $p<0.0001$ ), and 13.40 (95\% CI 7.64-23.51, $p<$ 0.0001 ) in Models 1,2 , and 3, respectively, which indicates that the serum creatinine level is a highly influential risk factor for anemia in patients with DM.

A high waist circumference showed significant ORs of 0.59 (95\% CI $0.44-0.79, p=0.0004$ ), 0.52 (95\% CI $0.31-$ $0.88, p=0.015$ ), and 0.47 (95\% CI $0.26-0.83, p=0.0097$ ) in Models 1, 2, and 3, respectively. Hypertriglyceridemia also showed significant ORs of 0.49 (95\% CI 0.34-0.70, $p<0.0001$ ), 0.69 (95\% CI 0.42-0.90, $p=0.0129$ ), and 0.62 (95\% CI 0.41-0.92, $p=0.0179$ ) in Models 1, 2, and 3 , respectively.

When the influence of risk factors on the prevalence of anemia among patients with DM was examined by sex, the results showed slight differences between men and women. Among men, the serum creatinine level, hypertension, and hypertriglyceridemia were significant factors in both the unadjusted (Model 1) and adjusted (Models 2 and 3) models. The serum creatinine level showed significant ORs of 10.31 (95\% CI 5.40-19.70, $p<0.0001$ ), 8.00 (95\% CI 3.84-16.65, $p<0.0001$ ), and 6.30 (95\% CI 3.08-12.90, $p<0.0001$ ) in Models 1,2 , and 3 , respectively. Hypertension also showed significant ORs of 2.49 (95\% CI 1.61-3.85, $p<0.0001), 2.14$ (95\% CI $1.31-3.49, p=0.0025)$, and 1.86 (95\% CI 1.18-3.13, $p=$
0.0191) in Models 1, 2, and 3, respectively. Additionally, hypertriglyceridemia showed significant ORs of 0.24 (95\% CI 0.13-0.43, $p<0.0001$ ), 0.38 (95\% CI 0.20-0.72, $p=0.003)$, and 0.40 (95\% CI 0.21-0.77, $p=0.0065)$ in Models 1, 2, and 3, respectively.

Among women, the serum creatinine level and a high waist circumference showed significant ORs in Models 1,2 , and $3(\mathrm{OR}=34.50,95 \% \mathrm{CI} 13.98-85.12, p<0.0001$ in Model 1; OR $=37.12$, 95\% CI 15.35-89.76, $p<0.0001$ in Model 2; and OR $=42.63,95 \%$ CI 17.25-105.36, $p<$ 0.0001 in Model 3). A high waist circumference alone showed significant ORs of 0.61 (95\% CI $0.42-0.89, p=$ $0.01), 0.49$ (95\% CI 0.28-0.87, $p=0.0149)$, and 0.38 (95\% CI $0.21-0.71, p=0.0022)$ in Models 1,2 , and 3 , respectively.

\section{Discussion}

The present study used nationally representative and highly reliable data from the KNHANES V, VI, and VII to investigate the association between DM and anemia among Korean adults aged $\geq 19$ years. Our findings indicated a clear association between DM and anemia among Koreans. In full adjusted Model 3, the OR for anemia in the DM group increased by 2.97 -fold as compared to the OR for the non-DM group. Accordingly, it was determined that the DM group had a more significant association with anemia than the non-DM group $(p<0.0001)$.

With regard to previous studies on DM and anemia, a study conducted among adults from the United States reported no association between DM and anemia, regardless of sex, which was in line with the conclusion drawn in a study from Spain that analyzed patients on nephrology dialysis also reported no association between DM and anemia [14, 21]. However, findings of the present study indicated an association between DM and anemia by sex, which is supported by findings from other studies on the association between iron-deficiency anemia and HbA1c level in DM' and the association between anemia and kidney function in patients with DM.' However, those studies used different diagnostic criteria for anemia and included participants of different ages and ethnic groups; thus, it is difficult to make direct comparisons between studies.

A previous Korean study [22] investigated the influence of DM on the onset of anemia among Korean adults, but the study found that after adjusting for other risk factors for anemia, DM was not an independent risk factor for anemia; this is contrary to the findings of the present study. Even when examining by sex, another Korean study [18] analyzed the OR of DM on the onset of anemia among Korean adults and found that DM increased the OR for anemia by 2.6 -fold among men after adjusting for age, education level, monthly household 
income, smoking status, alcohol consumption, iron intake, and disease history, whereas DM did not influence the onset of anemia among women.

The present study also investigated the influence of risk factors on the prevalence of anemia among patients with DM by sex. Our study found that men had a 5.66fold higher OR for anemia than women, and there was a significant association in interaction between sex and DM on the development of anemia (ORs of 0.39 in Model 3).

Recent studies have identified the associations of chronic kidney failure and elevated creatinine levels with metabolic syndrome, including DM [23]. The results showed that the serum creatinine level was a significant risk factor for anemia in both men and women. In particular, the serum creatinine level showed ORs of 6.30 $(p<0.0001)$ in men and $42.63(p<0.0001)$ in women after adjusting for demographic, lifestyle, disease, and health-related factors, which indicates that the serum creatinine level greatly influences the prevalence of anemia, especially among women with DM.

The association between serum creatinine level and anemia in patients with DM was a noteworthy finding in the present study. A previous study [24] found no significant association between serum creatinine level $(\mathrm{mg} / \mathrm{dL})$ and anemia among patients with DM, as well as no significant association with GFR $(\mathrm{mL} / \mathrm{min})$. In the present study, however, the serum creatinine level showed a strong association with anemia in women with DM. Such a difference could be attributed to the fact that the existing report was a case study that recruited patients with DM and had a sample size of only 142, whereas the present study analyzed data from 25,597 people. In addition, the existing study particularly recruited patients with type $2 \mathrm{DM}$, whereas the KNHANES included individuals with type 1 and type $2 \mathrm{DM}$. Additional studies are needed to investigate the mechanisms involved in DM, anemia, and kidney disease by sex.

The present study had some limitations because it used KNHANES data [25]. First, as the KNHANES was a cross-sectional survey study, the association between DM and anemia could be examined, although the causal relationship between DM and anemia must be interpreted with caution. Second, a major limitation in studying anemia is that the onset involves diverse and complex mechanisms; however, the KNHANES defined anemia simply based on the $\mathrm{Hb}$ level. Therefore, it was difficult to identify other risk factors for anemia such as vitamin $B_{12}$ level. Moreover, chronic anemia and irondeficiency anemia cannot be differentiated by the present study alone. Third, when defining the covariates hypertension and hyperlipidemia, this study defined only the numbers without considering whether participants were taking medication during the study period to lower their levels. Hypertension and hyperlipidemia are covariates rather than direct outcomes in this paper, and the two diseases are chronic diseases and belong to the related cardiovascular system. If each disease included people on medication, then it would be difficult to distinguish each group, which may have affected the significance of the results; therefore, people on medication were not considered. Another limitation of this study is that people whose diseases were being numerically controlled through drugs were not considered. Fourth, the number of patients with diabetic nephropathy among the included patients with DM could not be identified using the KNHANES data, making it difficult to accurately identify the association. Although the KNHANES provides nationally representative data, the survey itself did not focus on DM and anemia. As such, there is a lack of data on biological values such as those on the GFR and blood erythropoietin level, which could be used to determine kidney health [26].

While it is unclear whether correcting anemia is helpful for patients with DM, DM is known to cause various complications, such as diabetic nephropathy and cardiovascular disease, and increase hospitalization and mortality rates. Therefore, continued management and treatment of DM are important. Future studies should compare patients with kidney disease caused by DM and patients with DM who do not have kidney disease.

Our findings showed that, after adjusting for all other risk factors for anemia, DM was an independent risk factor for anemia among Korean men. Therefore, anemia should be monitored not only in women and malnourished patients but also in patients with DM. The management and treatment of anemia are important for preventing DM-related complications such as kidney disease and cardiovascular disease. Despite the limitations, the present study contributes to the existing literature in that it investigated the association between DM and anemia within the Korean population. This study is the first to investigate sex-based differences in factors that influence DM and anemia. Large-scale studies that overcome the limitations of the present study are needed in the future, and the findings of the present study may serve as baseline data.

\section{Conclusions}

Our findings indicate that there is an association between DM and anemia among Korean adults, with a strong association between DM and anemia among men. In addition, the serum creatinine level was identified as a factor that has a significant influence on DM and anemia among women. However, considering that the survey did not particularly investigate anemia, the mechanism could not be identified. As anemia may influence DM-related complications such as kidney disease and 
other cardiovascular diseases, management and treatment of anemia in patients with DM are considered highly important.

\section{Abbreviations}

BMI: Body mass index; Cl: Confidence interval; DM: Diabetes mellitus; FBS: Fasting blood sugar; GFR: Glomerular filtration rate; Hb: Hemoglobin; HbA1c: Glycated hemoglobin; HDL: High-density lipoprotein; KNHA NES: Korea National Health and Nutrition Examination Survey; OR: odds ratio

\section{Supplementary Information}

The online version contains supplementary material available at https://doi. org/10.1186/s12902-021-00873-9.

Additional file 1. Associations between DM and sex and interaction

between DM and sex in anemia

\section{Acknowledgements}

Not applicable.

\section{Authors' contributions}

MK, SL, and IH designed the study. MK and SL analyzed the data and wrote the manuscript. KP, EK, SY, and $I \mathrm{H}$ corrected the manuscript. All authors read and approved the final manuscript.

\section{Availability of data and materials}

The datasets generated and analyzed during the current study are available in the KNHANES repository (http://knhanes.cdc.go.kr). All data from the KNHA NES V, VI, and VII are coded and freely available.

\section{Declarations}

Ethics approval and consent to participate

The KNHANES V, VI, and VII were conducted by the Korea Centers for Disease Control and Prevention (KCDC). All survey protocols were approved by the institutional review board of the KCDC (approval numbers: 2013-07CON-034C, 2013-12EXP-03-5C, and 2015-01-02-6C). Informed consent was obtained from all participants when the surveys were conducted. Institutional review board approval was not required for the present study, as it did not deal with sensitive information and accessed only publicly available data from the KNHANES (JASENG IRB File No. 2019-03-006).

\section{Consent for publication}

Not applicable.

\section{Competing interests}

The authors declare that they have no competing interests.

\section{Author details}

'Jaseng Hospital of Korean Medicine, Seoul, Republic of Korea. ${ }^{2}$ Jaseng Spine and Joint Research Institute, Jaseng Medical Foundation, 3F JS Tower, 538 Gangnam-daero, Gangnam-gu, Seoul 06110, Republic of Korea. ${ }^{3}$ Department of Acupuncture \& Moxibustion, Dongguk University Bundang Oriental Hospital, Bundang, Republic of Korea. ${ }^{4}$ Department of Meridian and Acupoint, College of Oriental Medicine, Sang Ji University, Wonju, Republic of Korea.

Received: 8 June 2021 Accepted: 11 October 2021

Published online: 21 October 2021

\section{References}

1. Organization WH: Definition and diagnosis of diabetes mellitus and intermediate hyperglycaemia: report of a WHO/IDF consultation. 2006

2. Roglic G. WHO global report on diabetes: a summary. Int J Noncommun Dis. 2016;1(1):3. https://doi.org/10.4103/2468-8827.184853.

3. Organization WH: Global report on diabetes: World Health Organization. In: Geneva; 2016

4. Yeo S-J, Kim B-H. Effects of an intensive management program for diabetic patients on a blood biochemical profile and diabetes knowledge. Korean J
Community Nutr. 2018;23(2):148-61. https://doi.org/10.5720/kjcn.201 8.23.2.148

5. Association KD. Diabetes fact sheet in Korea 2016. Seoul: Korean Diabetes Association/Korea Centers for Disease Control and Prevention; 2012.

6. Association AD. Economic costs of diabetes in the US in 2012. Diab Care. 2013;36:1033-46 Diabetes Care 2013, 36(6):1797.

7. Silva T, Zanuzzi J, Silva C, Passos X, Costa B. Prevalence of cardiovascular diseases in diabetic and nutritional status of patientes. J Health Sci Instit. 2012:30(3):266-70

8. Irace C, Scarinci F, Scorcia V, Bruzzichessi D, Fiorentino R, Randazzo G, et al Association among low whole blood viscosity, haematocrit, haemoglobin and diabetic retinopathy in subjects with type 2 diabetes. $\mathrm{Br} J$ Ophthalmol. 2011;95(1):94-8. https://doi.org/10.1136/bjo.2009.172601.

9. Chaparro CM, Suchdev PS. Anemia epidemiology, pathophysiology, and etiology in low-and middle-income countries. Ann N Y Acad Sci. 2019; 1450(1):15-31. https://doi.org/10.1111/nyas.14092.

10. Kim SK, Kang HS, Kim CS, Kim YT. The prevalence of anemia and iron depletion in the population aged 10 years or older. Korean J Hematol. 2011; 46(3):196-9. https://doi.org/10.5045/kjh.2011.46.3.196.

11. McLean E, Cogswell M, Egli I, Wojdyla D, De Benoist B. Worldwide prevalence of anaemia, WHO vitamin and mineral nutrition information system, 1993-2005. Public Health Nutr. 2009;12(4):444-54. https://doi.org/1 $0.1017 /$ S1368980008002401.

12. Ezenwaka CE, Jones-LeCointe A, Nwagbara E, Seales D, Okali F. Anaemia and kidney dysfunction in Caribbean type 2 diabetic patients. Cardiovasc Diabetol. 2008;7(1):25. https://doi.org/10.1186/1475-2840-7-25.

13. BB HE, Xu M, Wei L, YJ GU, JF HAN, YX LIU, et al. Relationship between anemia and chronic complications in Chinese patients with type 2 diabetes mellitus; 2015

14. Astor BC, Muntner P, Levin A, Eustace JA, Coresh J. Association of kidney function with anemia: the third National Health and nutrition examination survey (1988-1994). Arch Intern Med. 2002;162(12):1401-8. https://doi.org/1 0.1001/archinte.162.12.1401.

15. Ahn S. Korean national health and nutrition examination survey. MS Thesis; 2005.

16. Lee B-W. Summary of the American Diabetes Association standards of medical Care in Diabetes 2016. J Korean Diab. 2016;17(2):79-82. https://doi. org/10.4093/jkd.2016.17.2.79.

17. Chung H, Moon H, Song B, Kim M. Hemoglobin, hematocrit and serum ferritin as markers of iron status. Korean J Nutr. 1991;24(5):450-7.

18. Kim J-H. The presence of diabetes mellitus and Anemia in Korean adultsbased on data from 2005 Korean National Health and nutrition examination survey (KNHANES III). J Nutr Health. 2008;41(6):502-9.

19. Levey AS, Stevens LA, Schmid CH, Zhang Y, Castro AF III, Feldman HI, et al. A new equation to estimate glomerular filtration rate. Ann Intern Med. 2009; 150(9):604-12. https://doi.org/10.7326/0003-4819-150-9-200905050-00006.

20. Expert Panel on Detection E. Executive summary of the third report of the National Cholesterol Education Program (NCEP) expert panel on detection, evaluation, and treatment of high blood cholesterol in adults (Adult Treatment Panel III). JAMA. 2001;285(19):2486.

21. Valderrábano F. Anaemia management in chronic kidney disease patients: an overview of current clinical practice. Nephrol Dial Transplant. 2002; 17(suppl_1):13-8.

22. Lee A-R, Yun J-M. Relationship between diabetes mellitus and Anemia in Korean adults-based on the Korean National Health and nutritional examination survey VI. J Korean Dietetic Assoc. 2017:23(1):54-63.

23. Chen N, Wang W, Huang Y, Shen P, Pei D, Yu H, et al. Community-based study on CKD subjects and the associated risk factors. Nephrol Dial Transplant. 2009;24(7):2117-23. https://doi.org/10.1093/ndt/gfn767.

24. Barbieri J, Fontela PC, Winkelmann ER, Zimmermann CEP, Sandri YP, Mallet EKV, et al. Anemia in patients with type 2 diabetes mellitus. Anemia. 2015; 2015:1-7. https://doi.org/10.1155/2015/354737.

25. Group NDD, Diabetes Nlo, Digestive, diseases K: diabetes in America: National Institutes of Health, National Institute of diabetes and digestive ... 1995

26. Ko EY, Kim SI, Jang YB, Min KH, Kim SH, Lee KS, et al. The clinical characteristics of Anemia in type 2 diabetic patients without overt nephropathy. J Korean Diab Assoc. 2004;28(5):425-31.

\section{Publisher's Note}

Springer Nature remains neutral with regard to jurisdictional claims in published maps and institutional affiliations. 